\title{
Sexually transmitted infections, sexual risk behaviours and perceived barriers to safe sex among drug users
}

\section{Mofizul Islam}

Australian Primary Health Care Research Institute, Australian National University, ACT

\section{Libby Topp}

Research Strategy Unit, Cancer Council NSW

\section{Katherine M. Conigrave \\ Drug Health Service, Royal Prince Alfred Hospital; Sydney Medical School, University of Sydney, New South Wales; National Drug and Alcohol Research Centre, University of New South Wales}

\section{Paul S. Haber \\ Discipline of Addiction Medicine, Sydney Medical School, University of Sydney, New South Wales}

\section{Ann White}

Redfern Harm Minimisation Clinic, Sydney Local Health District, New South Wales

\section{Carolyn A. Day}

Discipline of Addiction Medicine, Sydney Medical School, University of Sydney, New South Wales

n njecting drug users (IDUs) face dual risks for blood-borne virus transmission: through contaminated injecting equipment and risky sexual behaviours. ${ }^{1}$ Sexual transmission may act as a bridge between IDUs and other populations. ${ }^{2}$ Therefore, understanding sexual behaviours and barriers to safe sex practices, the prevalence of sexually transmitted infections (STI) and appropriate prevention interventions among IDUs is important. The use of alcohol and illicit drugs is associated with the spread of HIV and other STIs through risky sexual behaviours. ${ }^{3}$ Interventions to reduce sexual risk behaviours among IDUs have received less attention than interventions for reducing HIV risk associated with sharing contaminated injecting equipment. ${ }^{4}$
In the US, HIV prevalence among IDUs is $9 \%$ with considerable regional variation, ${ }^{5}$ and prevalence of both sexual risk behaviours and STIs among IDUs is also high. ${ }^{6}$ Little is known about sexual risk and STIs among Australian IDUs where HIV prevalence is $<2 \%$. $^{7}$ While high rates of sexual risk behaviours have been reported by Australian Needle and Syringe Program (NSP) Survey participants, ${ }^{7}$ the survey does not undertake STI screening. One Melbourne study has documented high prevalence of both sexual risk and moderate prevalence of STIs among a street-recruited sample of IDUs and recommended outreach screening to reduce the barriers to accessing sexual health services. ${ }^{8}$

\begin{abstract}
Objective: Drug users are at elevated risk of HIV and other sexually transmitted infections (STIs). This study examines prevalence of STIs and perceived barriers to safe sex among drug users accessing low-threshold primary healthcare in innercity Sydney.
\end{abstract}

Methods: Data were extracted manually from clients' medical records and analysed using STATA.

Results: Prevalence of HIV, syphilis, chlamydia and gonorrhoea were low (<2\%), whereas hepatitis C (62\%), hepatitis $A(30 \%)$, and previous exposure to hepatitis B (25\%) were more common. Recent unprotected vaginal and anal intercourse were reported by $85 \%$ and $26 \%$ of clients, respectively. Younger clients and those with a history of sex work or recent anal intercourse were more likely to report multiple recent unprotected sex partners. Having a regular sex partner was the most prevalent barrier to condom use (37\%), and was more likely to be identified by clients who were older, of Indigenous descent, and/or heterosexual. Drug intoxication was a second important barrier (20\%), and was more commonly identified by excessive alcohol users.

Conclusions: Targeted programs might increase awareness regarding the benefits of condom use and potential sexual risk associated with regular partners. Periodic assessments of alcohol use, and brief interventions for drug users who report problematic use, should also be considered.

Key words: injecting drug users, sexual risk behaviour, unprotected sex, lowthreshold healthcare

Aust NZ J Public Health. 2013; 37:311-15 doi: $10.1111 / 1753-6405.12077$ 
Low-threshold healthcare facilities endeavour to reduce major barriers IDUs face in accessing conventional healthcare facilities. They acknowledge risk behaviours without judgement or sanction and increase IDUs' likelihood of disclosing socially stigmatised behaviours. ${ }^{9110}$ Such services offer free condoms, safe sex education, STI screening, treatment and health promotion. Given the scant literature regarding prevalence of STIs and sexual risk among Australian IDUs, we aimed to investigate these among drug users attending such a service. Specifically, this report describes: (i) the prevalence of major STIs and self-reported sexual risk behaviours; (ii) correlates of sexual risk behaviours; and (iii) perceived barriers to safe sex among drug users.

\section{Method}

Data were derived from a validated client intake assessment form which is routinely filled in for every client accessing services from the Redfern Harm Minimisation Clinic. This service is an IDU-targeted low-threshold primary healthcare centre established in an existing NSP setting in inner-city, Sydney, Australia. ${ }^{11,12}$ This nurse-led facility provides primary healthcare on both 'drop in' and appointment bases. Referrals are received from other communitybased health services. Services include screening for bacterial STIs (mainly syphilis, gonorrhoea and chlamydia), blood-borne viral infection assessment for hepatitis C (HCV), HIV and hepatitis B, and referral to other appropriate healthcare centres and/or general physicians.

During initial presentation, nurses conduct a full assessment and record details about clients' demographic characteristics, drug use, risk behaviours related to blood-borne virus and STIs and other relevant health information. This assessment is updated annually for returning clients. Risk behaviours collected at the most recent assessment were recorded for this study. Further information was drawn from the medical files of clients diagnosed with an STI.

Perceived barriers to safe sex were documented through an openended question assessing reasons for not using condoms. Inter-rater reliability was high $(\kappa=0.92, p<0.01)$ between the two researchers who coded the first 100 client responses to this item.

\section{Pathological testing}

HIV was detected using enzyme immunoassay (HIV Combo AxSYM, Abbott Laboratories, USA) and positive specimens were subjected to Western blot confirmatory testing (HIV Blot 2.2, MP Diagnostics, Singapore). Syphilis was detected using enzyme immunoassay rapid plasma regain (VD Macro-vue RPR card test Vecton Dickinson), and chlamydia and gonorrhoea were detected using polymerase chain reaction (ProbeTec CT/GC assay from $\mathrm{BD})$ of urine samples. Roche Cobas Elecsys reagents were used for detecting IgG antibody of hepatitis A, HCV antibody and core antibody of hepatitis B. HCV antibody was further confirmed by enzyme immunoassay (Vitros Otrthoclinical HCV Blot 3.0 MP Diagnostics Germany), and qualitative HCV RNA by COBAS Ampliprep/COBAS AMPLICOR HCV Test version 2.0.

\section{Data collection and analysis}

The study was approved by the Sydney Local Health District Ethics Review Committee. Information on all 500 clients the clinic received between July 2006 and March 2011 was extracted manually from medical records by the first author. Multivariate logistic regression analysis derived adjusted odds ratios (AOR) and $95 \%$ confidence intervals (CI) controlling for variables associated at $p<0.15$ in univariate analysis to identify variables independently associated with: reporting recent (last 12 months) multiple unprotected sex partners (binary outcome: ' $\geq 2$ ' versus '0-1 partner') and correlates of perceived barriers to safe sex. Multicollinearity was assessed, and goodness-of-fit was examined using the HosmerLemeshow test. Data were analysed using STATA.

\section{Results}

Blood-borne virus and STI screenings were the main reasons for initial presentation; one-quarter of clients presented specifically for a sexual health assessment (Table 1). The prevalence of bacterial STIs (infectious chlamydia, gonorrhoea and syphilis) in the cohort was low $(<2 \%)$. One-quarter of clients reported two or more drugs of concern. Of the 385 clients who reported recent (preceding 12 months) unprotected sex, 44\% reported 2-5 unprotected-sex partners, $10 \%$ reported $6-10$ and $6 \%$ reported 11 or more. The most recent unprotected sexual event most often occurred in the preceding six months (93\%). Homosexual and bisexual clients were more likely than heterosexual clients to report unprotected anal intercourse ( $44 \%$ versus $24 \%, p<0.01$ ). Thirteen per cent reported a history of sex work, including 20 clients currently engaged in sex work. Prior sexual health screens were reported by 249 (57\%) clients, $19 \%$ of whom reported prior diagnoses and/or management of an STI.

\section{Correlates of unprotected sex with multiple partners and recent sexual risk behaviours}

Univariate analysis revealed younger ( $c$ older) clients (OR 1.05; CI 1.03-1.07), those with a history of sex work ( $\mathrm{OR}=2.3$; CI 1.3-4.1) and those who reported recent unprotected anal $\operatorname{sex}(\mathrm{OR}=3.3$; CI 2.15.4) were more likely than others to report recent multiple (two or more) unprotected-sex partners. Clients positive for $\mathrm{HCV}(\mathrm{OR}=0.54$; CI 0.34-0.80) or hepatitis B core antibody $(\mathrm{OR}=0.53$; CI 0.33-0.87), and those whose drug of concern was methamphetamine $(\mathrm{OR}=0.58$; $0.38-0.90)$, were less likely than others to report recent multiple unprotected-sex partners. Clients who reported unprotected sex less than one month prior were more likely than others to report multiple unprotected-sex partners ( $\mathrm{OR}=1.77$; CI 1.16-2.69). A linear trend was found between history of STI diagnosis and increasing number of unprotected sex partners in the preceding 12 months ( $p=0.03$ ). In multivariate analyses, younger age ( $\mathrm{AOR}=1.06$; $\mathrm{CI} 1.04-1.10)$, a sex work history $(\mathrm{AOR}=2.30$; $\mathrm{CI} 1.28-4.14)$ and unprotected anal sex $(\mathrm{AOR}=3.37$; $\mathrm{CI} 2.04-5.56)$ remained significantly associated with recent multiple unprotected-sex partners.

Aboriginal and/or Torres Straight Islanders were more likely than other participants $(p<0.05)$ to report an unprotected sexual episode 
Table 1: Demographic, drug use, sexual risk behaviours, blood-borne viral and STI-related information of 500 clients.

Variable

Mean age

(range, \pm SD)

Ethnicity

Aboriginal or Torres Strait Islanders Total sample

500

$(15-75 ; 9.03)$

Others

Gender

Male

Female

Transgender

Referral source

Residential alcohol and drug treatment agency

Co-located NSP

Self/family/friends

Other healthcare services

Primary reason for presentation ${ }^{\mathrm{b}}$

Blood-borne viral infection screening/vaccination $\quad 260$

Sexual health assessment/STI check-up $\quad 125$

Drug health related issues/injecting related injuries

Counselling/welfare services

General health issues

Sexual preference

Opposite gender

Same gender

Both genders

Route of drug administration

Injecting (IDU)

Not drug users

\section{Drug of concern}

Methamphetamine (ice)

Heroin

Alcohol

Current/past sex work

Yes

No

Unprotected anal sex in previous 12 months

Yes

No

Last unprotected penetrative sex

$<1$ month

$>1$ month

Clients underwent STIs and BBVIs tests

HIV

Syphilis RPR, EIA reactive

Gonorrhoea

Chlamydia 
within the preceding month. Compared to other men, men who expressed a preference for same-sex partners $(n=24)$ were more likely $(p=0.01)$ to report unprotected sex with multiple partners in the previous 12 months.

\section{Barriers to condom use}

Information about barriers to condom use was available for 293 clients, 89\% (261) of whom did not always use condoms (Table 2). Partner-related reasons were most common, with 96 clients (37\%) stating that they did not use condoms because they had regular or mostly regular partner(s). However, $27 \%$ of this group reported recent unprotected vaginal/anal sex with $\geq 2$ partners and another $4 \%$ with $\geq 6$ partners.

Subgroup analyses indicated that clients who reported having regular sex partners as the barrier to condom use were more likely than others to be older ( $\mathrm{AOR}=1.1, \mathrm{CI} 1.0-1.1)$, of Aboriginal/ Torres Strait Islander descent $(\mathrm{AOR}=3.3$, CI 1.3-8.4), and to prefer heterosexual sex $(\mathrm{AOR}=6.9, \mathrm{CI} 1.9-24.7)$. Respondents who

\section{Table 2: Clients' perceived barriers to condom use $(n=261)$.}

\section{Partner-related}

Regular or mostly regular/long-term partner (s)

Love/trust/familiarity with partners

Partner pressure/Partner may think I am not clean

Knew the partner (s) were clean/Felt safe with partner (s) 4

Trying to conceive/Partner is infertile

Regular partner deceased/ Otherwise partner may have unprotected sex with others

Female partner(s) only, therefore low-risk

Sex-work, difficult to always negotiate

Intoxication (drug-related)

Intoxication, drunk, stoned

Accepted offer for drug, craving for drug/Prolonged sex on drug

\section{Relating to condom}

Do not like condom/lack of sensation

Loss of spontaneity/ Interfere with continued erection

Condom break/slip/do not fit well/Allergy to condoms (latex)

Availability

Situational availability (not available at the required time)/ unplanned

Lack of access to condom/ Inconvenient to always carry condom

Spur of the moment/felt out of control

1

Percentages add up to greater than 100 , because some respondents state more than one barrier. reported alcohol as the drug of concern were more likely than others to identify intoxication as a barrier to condom use $(\mathrm{AOR}=2.8, \mathrm{CI}$ 1.5-5.1).

\section{Discussion}

Our findings indicate that prevalence of bacterial STIs and HIV is low among clients of this low-threshold service for IDUs, although prevalence of viral hepatic infections remains high. Our clients, like IDUs of other settings, tend to engage in high-risk sexual activity. ${ }^{13-15}$ However, as our data demonstrate, the client group is heterogeneous, which, together with reported rates of risk, demands a targeted intervention approach.

Consistent with the literature, unprotected sex was common among those who reported a regular partner. ${ }^{16}$ Of concern is that one-quarter of clients reported recent unprotected sex with $\geq 2$ partners. Clients' understandings of 'regular partner' are unclear, and clinical experience indicates that partnerships are often unstable among this client group. The risk of acquiring STIs is dependent upon individual behaviour, the structure of clients' sexual network and the prevalence and duration of infections in the network. ${ }^{17}$ Despite reported high-risk behaviours, the limited evidence available suggests that the prevalence of chlamydia, gonorrhoea and syphilis among IDUs in Australia is low, with a study among street-based IDUs in Melbourne documenting only slightly higher prevalence than we observed (chlamydia: $6 \%$ vs. $2 \%$; gonorrhoea: $1 \%$ vs. $0.5 \%$ and syphilis: $0 \%$ vs. $1.5 \%) .{ }^{8}$ Effective contact tracing procedures and treatment services in Australia ${ }^{17}$ may also play an important role in limiting average duration of infection and probability of transmission per sexual contact. Although this study did not collect information regarding the frequency of sexual activity, it is possible that reduced libido and drug-induced sexual dysfunction among IDUs ${ }^{19}$ may also lead to relatively low frequency of sexual activity.

Men who have sex with men (MSM) in our sample reported similar risk behaviours to gay periodic survey participants. For example, $65 \%$ of the $24 \mathrm{MSM}$ in our sample reported unprotected anal sex in the previous 12 months, similar to the $61.7 \%$ of 2010 Gay Periodic Survey participants who reported the same behaviour in the equivalent period.

Although not perfectly comparable, our data suggest lower prevalence of safe sex than among the general population of NSW, the Australian state in which this study was undertaken (1.3\% vs. $18 \%$, respectively, reported always using condoms). ${ }^{18}$ Similarly, half our clients reported recent unprotected sex with two or more partners, compared to $10 \%$ in the broader population. Our sample also reported a relatively high rate of unprotected anal sex. Although this was more commonly reported by homo/bisexual (40\%) than heterosexual clients (24\%), the practice was still relatively high in the latter group and this is consistent with previous reports among IDUs. ${ }^{5}$ Australia has, to date, managed to avert a generalised outbreak of HIV among IDUs, and HIV infection among Australian IDUs is largely confined to men who have sex with men. ${ }^{7}$ Earlier studies in other settings found that, despite the potential to play a 
preventive role, NSPs and other harm reduction programs have little effect on IDUs' safe sex practices, ${ }^{19}$ although sporadic initiatives have been implemented in recent years. ${ }^{20}$

Although most of our sample were polydrug users, clients who reported alcohol as a drug of concern were more likely than others to report intoxication as a barrier to condom use. A recent metaanalysis found that alcohol use is an independent risk factor for intentions to engage in unprotected sex. ${ }^{21}$ Regular assessments of alcohol use and targeted brief interventions at low-threshold services may improve clients' health outcomes.

The study is limited, as relevant information could not be collated for some clients. The sample was of consecutive clients; however it is not clear if clients of this service are representative of IDUs more generally. As identified above, information on the frequency of sexual encounters was not collected and low sexual activity may partially explain the low STI prevalence. Face-to-face interviews regarding sexual risk behaviours may also be subject to social desirability and other response bias. ${ }^{22}$

It is difficult to draw firm conclusions from these findings, given that just three bacterial STIs (infectious chlamydia, gonorrhoea and syphilis) were examined, and that the study did not assess sexual activity. Nevertheless, results suggest that the level of risk among this group is not as high as might be assumed. Further studies including the diagnosis of a broader range of STIs and the detailed characterisation of sexual activity would be useful to investigate this suggestion.

Despite the relatively low STI prevalence, the high prevalence of sexual risk behaviours indicates these clients remain at risk of STIs. Appropriately targeted programs might increase awareness among IDUs regarding the benefits of condom use and potential sexual risk associated with regular partners. The literature suggests that the efficacy of such interventions is variable across settings but, largely, IDUs reduce their sexual risk behaviours. ${ }^{20}$ Periodic assessments of alcohol use, and brief interventions for IDUs who report problematic use, should also be considered. Low-threshold services are an important avenue to provide such targeted programs and interventions. The attendance of IDUs at these facilities offers an important opportunity, as this group can be hard-to-reach and may prioritise their health lower than other competing demands they may face.

\section{Acknowledgements}

We thank our colleagues Sara and Steve for offering much needed services to the clients of Redfern Harm Minimisation Clinic.

\section{References}

1. Kral AH, Bluthenthal RN, Lorvick J, Gee L, Bacchetti P, Edlin BR. Sexual transmission of HIV-1 among injection drug users in San Francisco, USA: risk-factor analysis. Lancet. 2001;357(9266):1397-401.

2. Benotsch EG, Somlai AM, Pinkerton SD, Kelly JA, Ostrovski D, Gore-Felton $\mathrm{C}$, et al. Drug use and sexual risk behaviours among female Russian IDUs who exchange sex for money or drugs. Int J STD AIDS. 2004;15(5):343-47.

3. Topp L. Amphetamine-type stimulant use and risk-taking behaviours. In: Allsop $\mathrm{S}$, Lee N, editors. Perspectives on Amphetamine-type Stimulants. Melbourne (AUST): IP Communications; 2012. p. 69-82.

4. Strathdee SA. Sexual HIV transmission in the context of injection drug use: implications for interventions. Int J Drug Policy. 2003;14:79-81.

5. Centers for Disease Control and Prevention. HIV Infection and HIV-Associated Behaviors Among Injecting Drug Users - 20 Cities, United States, 2009. MMWR Morb Mortal Wkly Rep. 2012;61(8):133-8

6. Hwang LY, Ross MW, Zack C, Bull L, Rickman K, Holleman M. Prevalence of sexually transmitted infections and associated risk factors among populations of drug abusers. Clin Infect Dis. 2000;31(4):920-6.

7. Topp L, Day CA, Iversen J, Wand H, Maher L. Fifteen years of HIV surveillance among people who inject drugs: the Australian Needle and Syringe Program Survey 1995-2009. AIDS. 2011;25(6):835-42.

8. Bradshaw CS, Pierce LI, Tabrizi SN, Fairley CK, Garland SM. Screening injecting drug users for sexually transmitted infections and blood borne viruses using street outreach and self collected sampling. Sex Transm Infect. 2005;81(1):53-8.

9. Islam MM, Topp L, Iversen J, Day CA, Conigrave KM, Lisa Maher on behalf of the Collaboration of Australian NSPs. Healthcare utilisation and disclosure of injecting drug use among clients of Australia's needle and syringe programs. Aust N Z J Public Health. 2013;37(2):148-54

10. Rich JD, McKenzie M, Macalino GE, Taylor LE, Sanford-Colby S, Wolf F, et al. A syringe prescription program to prevent infectious disease and improve health of injection drug users. J Urban Health. 2004;81(1):122-34.

11. Islam MM, Grummett S, White A, Reid SE, Day CA, Haber PS. A primary healthcare clinic in a needle syringe program may contribute to HIV prevention by early detection of incident HIV in an injecting drug user. Aust NZJ Public Health. 2011;35(3):294-5.

12. Islam MM, Topp L, Conigrave KM, White A, Haber PS, Day CA. Are primary health care centres that target injecting drug users attracting and serving the clients they are designed for? A case study from Sydney, Australia. Int J Drug Policy. 2012 July 18 PMID: 22818978.

13. Zhao M, Du J, Lu GH, Wang QY, Xu H, Zhu M, et al. HIV sexual risk behaviors among injection drug users in Shanghai. Drug Alcohol Depend. 2006;82 Suppl 1:S43-7.

14. Tyndall MW, Patrick D, Spittal P, Li K, O'Shaughnessy MV, Schechter MT. Risky sexual behaviours among injection drugs users with high HIV prevalence: implications for STD control. Sex Transm Infect. 2002;78 Suppl 1:1170-5.

15. Gyarmathy VA, Li N, Tobin KE, Hoffman IF, Sokolov N, Levchenko J, et al. Unprotected sex in heterosexual partnerships of injecting drug users in st. Petersburg, Russia. AIDS Behav. 2011;15(1):58-64.

16. Bogart LM, Kral AH, Scott A, Anderson R, Flynn N, Gilbert ML, et al. Sexual risk among injection drug users recruited from syringe exchange programs in California. Sex Transm Dis. 2005;32(1):27-34.

17. McCarthy M, Haddow LJ, Furner V, Mindel A. Contact tracing for sexually transmitted infections in New South Wales, Australia. Sex Health. 2007;4(1): 21-5

18. Rissel C, Bauman A, McLellan L, Lesjak M. A 1994 population survey of the number of sexual partners over a 12-month period in New South Wales, Australia. Venereology. 2000;13(3):111-17.

19. Rhodes T, Quirk A. Where is the sex in harm reduction? Int J Drug Policy. 1995;6:76-81.

20. DesJarlais DC, Semaan S. Interventions to reduce the sexual risk behaviour of injecting drug users. Int J Drug Policy. 2005;16S:S58-S66.

21. Rehm J, Shield KD, Joharchi N, Shuper PA. Alcohol consumption and the intention to engage in unprotected sex: systematic review and meta-analysis of experimental studies. Addiction. 2011;107:51-9.

22. Islam MM, Topp L, Conigrave KM, Beek Iv, Maher L, White A, et al. The reliability of sensitive information provided by injecting drug users in a clinical setting: clinician-administered versus audio computer-assisted self-interviewing (ACASI). AIDS Care. 2012;24(12):1496-503 